\title{
Extreme Environment Refractometers designed in Integrated Optical Fibre
}

\author{
Christopher Holmes, Alexander Jantzen, Stephen G Lynch, Paul C Gow, Teresa I Ferreiro, \\ Peter GR Smith \\ University of Southampton, Southampton, SO17 1BJ, UK
}

Integrated Optical Fibre (IOF) is a passive optical platform that directly integrates optical fibre to a planar substrate, illustrated in Fig 1(a). It possess advantages associated with optical fibre such a low propagation loss, whilst enabling planar functionality commonplace with integrated optics. Planarization is uniquely achieved through a modified Flame Hydrolysis Deposition (FHD) technique that forms a robust glass alloy between the fibre and substrate. The binding medium is of optical quality and resistant against common solvents, chemicals and elevated temperatures of up to $1000^{\circ} \mathrm{C}$. Furthermore as fibre can be brought seamlessly on-off chip there is no need for glues or cumbersome coupling arrangements, which are typically a point of mechanical weakness when monitoring harsh environments.

Thus far, developments in IOF have largely utilised either the environmental stability [1] and/or physical robustness [2] of the platform and not the optical quality of the binding medium. This work reports for the first time and evanescent field based refractometer using IOF, in which the optical mode directly interacts with the deposited FHD glass and the external environment. The concept for design is based largely on so termed thinnedFBGs [3], in which an optical fibre is wet etched using buffered HF. Through applying a similar geometry to the IOF format one can achieve external refractive index sensitivity, but with an enhanced mechanical integrity and capacity for integration.
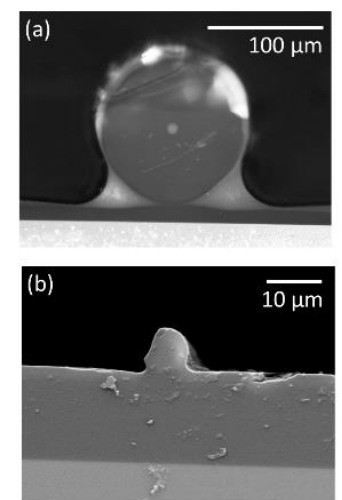

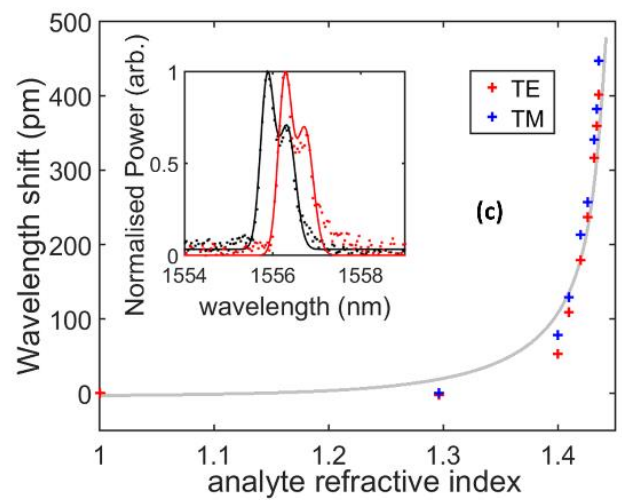

Fig. 1 (a) a cross-section profile of an Integrated Optical Fibre with SMF-28. (b) A cross-section profile of an Integrated Optical Fibre with a wet etched SMF-28 fibre (fibre $8 \mu \mathrm{m}$ in diameter). (c) The optical response of a Bragg grating in construct (b), under exposure to Cargille refractive index matching liquids.

This work reports a proof-of-concept refractometer design, fabricated through wet etching SMF-28 fibre in a buffered HF solution then applying it in an IOF format. For the device depicted in Fig 1 (b), etching is continued until a diameter of $8 \mu \mathrm{m}$ is reached. This etched fibre is then subsequently transferred and layered-up onto a silicon wafer that has a thick thermally grown oxide $(15 \mu \mathrm{m})$. The purpose of the oxide is to act as an optical buffer layer (clad), ensuring the final device maintains a guided mode. To form the IOF and maintain as much evanescent field exposure to the external environment a $0.5 \mu \mathrm{m}$ FHD layer is deposited and consolidated at $1250^{\circ} \mathrm{C}$, leaving the cross sectional geometry shown in Fig 1 (b). The FHD recipe is a silicate, doped with boron and phosphorus that once consolidated has a refractive index of 1.4452 (at a wavelength of $1553 \mathrm{~nm}$ ).

Changes in external refractive index were inferred through use of a Direct UV Written fibre Bragg grating [2]. The grating was inscribed at a fluence of $30 \mathrm{kJcm}^{-2}$ into the fibre core, after the FHD had been consolidated. To enhance UV photosensitivity the chip was hydrogen loaded at 120 bar for 7 days. Cargille refractive index matching liquids (series AA and AAA) were used to calibrate the index response illustrated in Fig 1(c). At refractive indices approaching 1.45, a spectral sensitivity of $32 \mathrm{~nm} /$ riu is measured. It is understood that further enhancement can be made through optimising fibre diameter, FHD refractive index, writing fluence and planar processing enhancements. The latest sensitivity results will be presented along with an analysis as well as operational responses in harsh environments including exposure and interrogation of Jet A1.

\section{References}

[1] S. G. Lynch, C. Holmes, S. A. Berry, C. James, A. Jantzen, T. I. Ferreiro, and P. G. R. Smith, "External cavity diode laser based upon an FBG in an integrated optical fiber platform," Opt. Ex., 24, 8, pp. 22781-22788, (2016).

[2] C. Holmes, J. C. Gates, and P. G. R. Smith, "Planarised optical fiber composite using flame hydrolysis deposition demonstrating an integrated FBG anemometer," Opt. Express, 22, 26, p. 32150, (2014).

[3] A. Iadicicco, A. Cusano, S. Campopiano, 'Thinned Fiber Bragg Gratings as Refractive Index Sensors', IEEE Sensors Journal, 5, 6, (2005). 